\title{
Are donor lymphocytes a barrier to transplantation tolerance?
}

Jawaher Alsughayyir ${ }^{1,2}$ PhD, Reza Motallebzadeh M.D, FRCS ${ }^{3}$, Gavin J. Pettigrew ${ }^{1 *}$ M.D, FRCS

${ }^{1}$ University of Cambridge, School of Clinical Medicine, Cambridge, UK, CB2 0QQ.

${ }^{2}$ King Saud University, College of Applied Medical Science, Riyadh, Saudi Arabia²King Saud University

${ }^{3}$ University College London, UK, NW3 2PF; ${ }^{4}$ Department of Nephrology \& Transplantation, Royal Free London NHS Foundation Trust, London, UK, NW3 2QG

\section{*Corresponding author}

Mr. Gavin J. Pettigrew.

University of Cambridge

School of Clinical Medicine

Box 202, Level E9

Addenbrookes Hospital

Cambridge

CB2 OQQ

UK

Telephone 01223336975

Fax 01223762523

E-mail gjp25@cam.ac.uk 


\begin{abstract}
:
Purpose of review:

Following solid organ transplantation, populations of donor lymphocytes are frequently found in the recipient circulation. Their impact on host alloimmunity has long been debated but remains unclear, and it has been suggested that transferred donor lymphocytes may either promote tolerance to the graft, or hasten its rejection. We discuss possible mechanisms by which the interaction of donor passenger lymphocytes with recipient immune cells may either augment the host alloimmune response, or inhibit it.
\end{abstract}

\title{
Recent findings:
}

Recent work has highlighted that donor T lymphocytes are the most numerous of the donor leukocyte populations within a solid organ transplant and that these may be transferred to the recipient after transplantation. Surprisingly, graft-versus-host recognition of MHC class II on host B cells by transferred donor CD4 T cells can result in marked augmentation of host humoral alloimmunity and lead to early graft failure. Killing of donor CD4 T cells by host NK cells is critical in preventing this augmentation.

\section{Summary:}

The ability of passenger donor CD4 T cells to effect long-term augmentation of the host humoral alloimmune response raises the possibility that ex-vivo treatment or modification of the donor organ prior to implantation may improve long-term transplant outcomes.

Keywords: Tolerance, allorejection, unlinked-help, GVHD, alloantibody, donor lymphocytes 


\section{Introduction}

In solid organ transplantation (SOT), the delivery of passenger donor lymphocytes (DLs) to recipients is almost unavoidable. Passenger lymphocytes have been detected in the recipient's circulation within the first two hours following murine liver ${ }^{1}$, primate kidney ${ }^{2}$; and human liver transplantation ${ }^{3,4}$. The presence of DLs can be associated with graft versus host disease (GVHD), passenger lymphocyte syndrome, or may possibly induce tolerance. The impact of transfer of DLs will likely be determined by: the microenvironment of the transplanted organ; migration and sensitisation status of DLs; and the capacity for interaction with host immune constituents, as recently reviewed ${ }^{5}$.

Organs such as the small intestines and lungs represent the first physiological mucosal defence barrier and possess large numbers of graft-resident lymphocytes. The generally poor long-term outcomes associated with these organs may be attributed to the simultaneous transfer of DLs contained within the allograft upon transplantation ${ }^{6}$. Tissueresident lymphocytes have been a particular research focus recenty, but the role of this subset in SOT has yet to be clarified. This review will first describe the make-up of the tissueresident lymphocyte populations that are likely to be present within an allograft. We will then discuss how these populations may contribute to graft tolerance and rejection, before considering how strategies that specifically target DLs may be used to prolong graft survival.

\section{Passenger lymphocytes populations within an allograft.}

The nature of tissue-resident lymphocytes differs in lineage and phenotype and includes B cells ${ }^{7}, \mathrm{CD} 8+{ }^{89}$, FoxP3 $+{ }^{10,11}$, innate $\gamma \delta^{12,13}$, NK T cells ${ }^{14} 15$ and CD4 T cells ${ }^{16}$ which have been reported in various tissues such as the skin, gut, lungs, kidney, and liver. To what extent these populations remain resident within the allograft or egress to the recipient's 
circulation is not yet known, but is clearly an important distinction - those DLs that migrate to the recipient's secondary lymphoid tissue will presumably have greater influence on host alloimmunity. In this respect, this review will focus on donor B cells and CD4+ T cells, because their potential for migration and interaction with host immune constituents suggests the greatest capacity for shaping the alloimmune response.

\section{Passenger donor B lymphocytes in allograft rejection and tolerance.}

Although not as prominent feature as T lymphocytes, circulating donor -strain B lymphocytes ( $\mathrm{dnB}$ cells)are frequently detectable in recipients of solid organ transplants, particularly following liver and intestinal transplantation ${ }^{17}$. Appreciable numbers are also released into the circuit during ex vivo normothermic perfusion of porcine kidneys ${ }^{18}$. How such transfer impacts upon the host alloimmune response remains unclear. Most immediately, migrating dnB cells will deliver MHC class I and class II alloantigen, and could potentially prime cytotoxic CD8 T cell and direct-pathway CD4 T cell alloresponses in the recipient. However, resting B cells do not generally express sufficient co-stimulatory ligands for activation of naïve T cells ${ }^{19-21}$, and indeed, have been targeted as a possible therapeutic strategy in autoimmunity ${ }^{22}$, raising the possibility that transferred dnB cells may inhibit the host response against the graft. Against this, activated ${ }^{23,24}$ or memory ${ }^{25,26}$ B cells are effective antigen presenting cells for driving naïve T cell activation, and it seems likely that even if transferred as naïve cells, exposure to previously unencountered recipient antigen within the inflammatory environment of the graft ${ }^{27}$, will lead to full B cell activation. In support, recent murine studies have reported a positive role for passenger dnB cells in triggering recipient $\mathrm{CD} 4 \mathrm{~T}$ cell alloimmunity ${ }^{28}$, although the impact was relatively modest when compared to the contribution from transferred donor dendritic cells. 
One striking manifestation of the transfer of dnB cells is Passenger Lymphocyte Syndrome, in which haemolysis is triggered by donor B cell recognition of mismatched $A B O$ blood group antigens in the recipient ${ }^{29}$. Whether this relates to transfer of plasma cells or to B cells that undergo further differentiation to antibody secretors within the recipient is not known, but the latter is suggested by a recent report highlighting similar donor-derived antibody profiles in a pair of recipients from the same deceased kidney donor ${ }^{30}$. The recipient antibody responses observed with Passenger Lymphocyte Syndrome appear transient, presumably because transferred donor plasma cells are not deposited in an appropriate recipient niche to facilitate long-term survival, and it is therefore doubtful that they contribute to allograft rejection, particularly because transfer of responsiveness against donor HLA antigen would be highly unusual.

Several recent clinical studies have suggested that a state of operational tolerance is associated with a signature B cell phenotype ${ }^{31-33}$, with an expanded transitional B cells population. Although suppressor function has not been confirmed in the clinic setting, this is consistent with development of regulatory $B$ cells ${ }^{34}$, and B cells have been shown to be important mediators of tolerance in murine transplant models ${ }^{35}$. It is generally assumed that the putative regulatory B cell population is of recipient origin, and is seems unlikely, given their presentation of mismatched MHC class I and class II alloantigens, that donor B cells would survive sufficiently long in the recipient to provide regulatory function. However, although poorly understood, regulatory B cell development is favoured by an inflammatory environment ${ }^{36,37}$, and requires positive signalling via the $B$ cell receptor, CD40 and TLR ligation ${ }^{38}$. Thus although not yet demonstrated, Interactions with host $\mathrm{T}$ cells and antigen presenting cells could conceivably provide the appropriate triggers for differentiation of 
migrating passenger donor B lymphocytes into regulatory cells that promote allograft survival.

\section{Passenger donor T lymphocytes in allograft rejection and tolerance}

The most abundant lymphocyte subset contained within a SOT is likely to be the CD8 and CD4 $T$ cell ${ }^{16,18}$. While these may be naïve circulating $T$ cells that are trapped within the graft microcirculation, additional populations of memory lymphocytes within the graft parenchyma (so called tissue resident memory ( $T_{R M}$ ) lymphocytes) are also likely to be transferred (cite the Buero and Turner review papers). Transfer of donor T lymphocytes ( $\mathrm{dnT}$ cells) is most readily manifest by the development of graft versus host disease in the recipient ${ }^{39,} 40$.

One might anticipate that GVH responses mediated by dnT cell transfer would favour enhanced allograft survival, by inhibiting host alloreactivity through cytotoxic destruction of host antigen presenting cells and alloreactive lymphocyte subsets. Our studies of mouse chronic heart allograft rejection however highlighted that in the MHC class II mismatched 'bm12' to C57BL/6 model, a key component of the rejection response was the triggering of recipient anti-nuclear autoantibody responses ${ }^{41,42}$. These responses were class-switched, but surprisingly, $T$ cell help for their initiation was provided, not by recipient CD4 T cells, but by donor CD4 T cells. The potential contribution of autoimmunity to allograft rejection is increasingly emphasised (reviewed in ${ }^{43-45}$ ) and T cell depletion experiments suggested a direct role for the autoantibody response observed in our model in mediating progression of allograft vasculopathy ${ }^{41,46}$

Autoantibody responses did not develop in mice that selectively lacked MHC class II expression only on B cells, highlighting that cognate interaction between the dnCD4 T cell 
and host B cell was the direct trigger for initiating humoral autoimmunity. This raises the question as to the precise MHC II / peptide complex that was recognised on the surface of the host B cells, and in this regard it was notable that the entire follicular B cell population up-regulated MHC class II and costimulatory ligand expression, in keeping with global activation $^{46}$. Further experiments incorporating $T$ cell deficient recipients highlighted that the critical step for recipient B cells differentiation to an antibody-secreting cell was concurrent BCR ligation with target antigen ${ }^{46}$. Because of the peculiarities of direct-pathway allorecognition, the dnCD4 T cell fraction is likely to recognise the majority, if not all, MHC class II complexes of host B cells, irrespective of the particular peptide bound in the binding groove. Thus, the dnCD4 T cells provide permissive help to all B cells; antibody secretion is determined instead by availability of target antigen (figure 1a). Presumably, the autoantibody responses observed in our experiments reflect activation of anergic autoreactive B cells that are already bound via their BCR to target autoantigen.

An intriguing possibility raised by this unusual, 'peptide-degenerate' help is that dnCD4 T cells can provide help to recipient B cells for the production of alloantibody against alloantigenic determinants expressed on the surface of the dnCD4 T cell (figure 1b). This occurs despite those CD4 T cells clearly being tolerant to that antigen when encountered restricted to self in the donor ${ }^{47}$. We further showed that the provoked alloantibody response resulted in rapid lysis of dnCD4 T cell fraction; effectively the dnCD4 T cells actively promote their own destruction. This further suggests that passenger CD4 T cells within could contribute to graft rejection. This was tested by developing a murine model of chronic heart allograft rejection in which the donor 'bm12.Kd.IE' strain differed from the C57BL/6 recipient at additional MHC class I $\left(\mathrm{H}-2 \mathrm{~K}^{\mathrm{d}}\right)$ and class II (I-E and I-A $\left.{ }^{\mathrm{bm} 12}\right)$ loci. Heart grafts 
were rejected slowly, with the development of progressive allograft vasculopathy that was associated with robust alloantibody responses directed against the mismatched class I and class II alloantigens. Critically, alloantibody responses were markedly reduced in recipients of heart grafts from T-cell depleted donors, as were recipient cytotoxic CD8 T cell alloimmune and indirect-pathway CD4 T cell responses. Heart allografts from T cell deficient donors exhibited markedly reduced allograft vasculopathy ${ }^{47}$, thus confirming an important role for passenger dnCD4 T cells in augmenting host alloimmunity.

Although these findings would appear to shift emphasis from the recipient alloreactive CD4 T cell population as the central mediator of allograft rejection, it was notable that rejection was still dependent upon the recipient CD4 T cell fraction. Our ongoing work suggests that recipient CD4 T cells are required for provision of essential T follicular helper cell function that maintains long-lasting germinal centre alloantibody responses: although dnCD4 T cells could still trigger recipient humoral immunity in T cell deficient recipients, germinal centre responses did not develop and heart grafts survived indefinitely without development of allograft vasculopathy ${ }^{46}$. Thus optimal recipient effector humoral responses required collaboration between donor and recipient CD4 T cell fractions (figure $1 \mathrm{c}$ ).

The model we employed was undoubtedly developed to facilitate examination of the potential contribution of dnCD4 T cells to graft rejection, and could be justly criticised as lacking immediate clinical relevance. What therefore are the likely implications of our findings to clinical transplantation? Firstly, our models were characterised by relatively limited MHC mismatch between the donor and recipient and the ability of donor CD4 T cells to interact productively with the recipient B cell population was dependent upon avoidance of rapid destruction by host NK cell recognition. Notably, in a completely MHC-mismatched 
(BALB/C to C57BL/6) model of chronic alloantibody mediated rejection, augmentation of the host alloantibody response by passenger CD4 T cells only occurred upon depletion of the recipient NK cell population. Such depletion resulted in rapid (acute) graft rejection ${ }^{47}$. The role of host NK cells in graft rejection remains controversial ${ }^{48}$, and our findings highlight an important, and previously unappreciated, role for NK cells in preventing graft rejection through recognition of donor passenger lymphocytes. Killer cell Immunoglobulin-like receptor (KIR) recognition is complex and evolving ${ }^{49}$, but current $\mathrm{MHC}$ matching practices in clinical renal transplantation will result in approximately half of donor - recipient combinations being matched at KIR loci ${ }^{50}$, thereby enabling donor passenger lymphocytes to avoid rapid NK-cell mediated destruction. Whether this results in poorer long-term allograft survival is not known, because studies that have examined the role of NK cells in clinical graft rejection have generally studied MHC-matched donor / recipient combinations to avoid the confounding impact of adaptive HLA allorecognition ${ }^{50,51}$ whereas the impact of NK cell recognition observed in our model is dependent upon donor / recipient MHC class II mismatching. Avoidance of NK cell killing does not, however, guarantee long-term survival of the donor CD4 T cell fraction; as mentioned above, the donor CD4 T cell fraction was still killed rapidly (within one week of transplantation) by the adaptive alloimmune responses that they provoke in the host. This illustrates that short-lived immune interactions that occur in the peri-transplant period may have long-lasting consequences; despite their rapid destruction, the impact of donor CD4 T cells on augmenting host humoral alloimmunity was evident many weeks after transplantation.

Most solid organs are now known to harbour populations of resident memory $T\left(T_{R M}\right)$ lymphocytes (reviewed in ${ }^{52}$ ), which are phenotypically distinct from the central and effector 
memory subsets. Donor-derived $T_{\mathrm{RM}}$ lymphocytes will presumably be transferred within most solid organ allografts, yet their contribution to host alloimmunity is not known ${ }^{5,53}$. Although our experiments did not distinguish between $\mathrm{T}_{\mathrm{RM}}$ lymphocytes within graft parenchyma and naïve circulating T cells caught in the graft microcirculation, they do nevertheless suggest a potential mechanism by which transferred donor $T_{\text {RM }}$ cells may influence the host alloimmune response. In this respect, it was notable that heart allografts from donors that had been primed six weeks earlier by a recipient strain skin graft (thus generating anti-recipient memory responses) provoked stronger alloantibody responses and were rejected much more rapidly than heart grafts from naïve donors ${ }^{46,47}$.

Our results suggest that strategies that deplete passenger donor lymphocytes within the allograft may hold potential to prolong allograft survival. This could possibly be achieved by performing ex vivo normothermic perfusion of the organ after retrieval; an approach that is gaining popularity because of its potential to improve 'recondition' the organ and improve viability ${ }^{54}$. Early reports suggest that substantial numbers of donor T lymphocytes are recovered from the circuit during the ex-vivo perfusion phase ${ }^{18}$, that would otherwise be released into the recipient's circulation.

Before such strategies are attempted, however, it is worth considering whether resident donor lymphocytes could provide protective function and promote graft survival. This has not been studied, but mucosal $\mathrm{T}_{\mathrm{RM}}$ cells provide an important first-line defence against tissue re-infection, and are likely involved in maintaining cytomegalovirus (CMV) latency ${ }^{55-}$ 57. Deletion of donor TRM within an allograft could therefore possibly exacerbate the intragraft inflammatory milieu due to CMV re-activation, which may in turn provoke host alloimnunity and increase the incidence of allograft rejection ${ }^{58}$. 
In addition to $T_{R M}$ lymphocytes, there is increasing evidence supporting a distinct population of tissue-resident regulatory T cells ${ }^{59}$. These are thought to regulate local responses within the organ and suppress development of autoimmunity - T cell receptor sequencing suggests skewing towards recognition of self-antigen ${ }^{60,61}$. Persistence of tissue-resident donor regulatory $T$ cells after transplantation could conceivably favour allograft survival, through, for example, production of suppressor cytokines, such as IL-10 and TGF- $\beta$, that promote an anti-inflammatory environment within the allograft. This has not been tested, but resonates with the growing appreciation that local immune events within the graft shape the recipient's alloimmune response ${ }^{62,63}$, and parallels the observation that recipient regulatory T cells mediate suppression principally within the allograft ${ }^{64,65}$.

Finally, similar to the peptide-degenerate, graft-versus-host recognition of recipient MHC class II complexes by donor-strain CD4 T effector cells, passenger donor CD4 T regulatory cells may also interact with a large proportion of the recipient B cell population. This interaction would instead, however, be expected to profoundly inhibit host humoral immunity (figure $1 \mathrm{~d}$ ). In support, bm12 heart allografts from donors that have been depleted of regulatory T cells (by administering anti-CD25 mAb to the bm12 donor prior to heart allograft rejection) are rejected much more rapidly by C56BL/6 recipients than heart grafts from unmodified bm12 donors (unpublished data, GJP).

\section{Conclusions}

Ex vivo perfusion studies suggest that the T lymphocytes is the most dominated subset. Whether this reflects release of circulating $\mathrm{dnT}$ cells or mobilisation of $\mathrm{T}_{\mathrm{RM}}$ cells from graft parenchyma has yet to be determined. The mechanism by which dnCD4 T cell interact with the recipient $B$ cells to was highlighted in our work, where rejection of murine heart 
allografts from donors primed against recipient was accelerated. A testable clinical consequence of this observation is that kidney transplants from living donors sensitised against recipient (typically mother to offspring) may be associated with higher rates of rejection than normal.

\section{Key points}

- Transfer of passenger donor lymphocytes is common after solid organ transplantation

- Transferred passenger donor CD4 T cells can potentially interact productively with recipient $B$ cells for long-lasting augmentation of the host alloimmune response.

- This augmentation is more pronounced if memory donor CD4 T cells with specificity for recipient are transferred.

- Host NK cell recognition and destruction of passenger lymphocytes is critical for preventing this interaction between donor and recipient lymphocytes. 


\section{Acknowledgements}

None

\section{Financial support and sponsorship}

This work was supported by a British Heart Foundation project grant, the National Institute of Health Research Cambridge Biomedical Research Centre and the National Institute of Health Research Blood and Transplant Research Unit.

\section{Conflicts of interest}

The authors declare no conflict of interest 


\section{Figure 1}

Heading: Proposed peptide-degenerate help signals provided by donor CD4 T cells to recipient $B$ cells.

Legend: a) Top panel: i) Donor CD4 T cells TCR recognise all MHC class II expressed on recipient $B$ cells as foreign regardless of the loaded peptide in a peptide-degenerate manner which result in global activation of all recipient $B$ cells. However, recipient $B$ cells differentiation into antibody-secreting plasma cells is determined by the concurrent BCR ligation to their target antigens (ii). Therefore, recipient $B$ cells differentiate into plasma cells that release alloantibodies (iii). Bottom panel: Cognate interaction between TCR on donor CD4 T cells and MHC class II on recipient B cells in peptide-degenerate manner (i) can cause $B$ cell priming; however, if $B C R$ s on primed recipient $B$ cells do not bind their corresponding antigen (ii), $B$ cells would not differentiation to plasma cells.

b) Donor CD4+ $\mathrm{T}$ cells expressing alloantigenic determinants that are recognised by recipient $B$ cells (i) can induce activation to recipient B cells in the same peptide-degenerate manner described in panel (a), leading to donor-specific alloantibody production that eventually leads to dnT cell lysis (ii).

c) Donor CD4+ T cells alloresponse is triggered by GVHD allorecognition (i), the provision of help from recipient CD4 T cells (ii) is essential to their subsequent differentiation into $T_{F H}$ CD4 T cells ( $\mathrm{RCT}_{\mathrm{FH}}$ ) for maintenance of GC humoral autoimmunity (iii).

d) Donor CD4+ Treg cells can recognise MHC class II on all recipient B cells regardless of presented-peptide specificity, and profoundly suppress host humoral immunity. 
References:

1. Tay SS, Lu B, Sierro F, Benseler V, McGuffog CM, Bishop GA, et al. Differential migration of passenger leukocytes and rapid deletion of naive alloreactive CD8 T cells after mouse liver transplantation. Liver Transpl. 2013; 19(11): 1224-35.

2. Nadazdin O, Boskovic S, Wee SL, Sogawa H, Koyama I, Colvin RB, et al. Contributions of direct and indirect alloresponses to chronic rejection of kidney allografts in nonhuman primates. J Immunol. 2011; 187(9): 4589-97.

3. Starzl TE, Demetris AJ, Trucco M, Ramos H, Zeevi A, Rudert WA, et al. Systemic chimerism in human female recipients of male livers. Lancet. 1992; 340(8824): 876-7.

4. Starzl TE, Demetris AJ, Murase N, Ildstad S, Ricordi C, Trucco M. Cell migration, chimerism, and graft acceptance. Lancet. 1992; 339(8809): 1579-82.

5. Turner DL, Gordon CL, Farber DL. Tissue-resident T cells, in situ immunity and transplantation. Immunol Rev. 2014; 258(1): 150-66.

6. Lodhi SA, Lamb KE, Meier-Kriesche HU. Solid organ allograft survival improvement in the United States: the long-term does not mirror the dramatic short-term success. Am J Transplant. 2011; 11(6): 1226-35.

7. Sathaliyawala T, Kubota M, Yudanin N, Turner D, Camp P, Thome JJ, et al. Distribution and compartmentalization of human circulating and tissue-resident memory T cell subsets. Immunity. 2013; 38(1): 187-97.

8. Steinert EM, Schenkel JM, Fraser KA, Beura LK, Manlove LS, Igyarto BZ, et al. Quantifying Memory CD8 T Cells Reveals Regionalization of Immunosurveillance. Cell. 2015; 161(4): 737-49.

*9. Mackay LK, Minnich M, Kragten NA, Liao Y, Nota B, Seillet C, et al. Hobit and Blimp1 instruct a universal transcriptional program of tissue residency in lymphocytes. Science. 2016; 352(6284): 459-63.

Identified Hobit-Blimp1 as a universal transcription module that instructs tissue retention in diverse tissue-resident lymphocyte population.

10. Kolodin D, van Panhuys N, Li C, Magnuson AM, Cipolletta D, Miller CM, et al. Antigen- and cytokine-driven accumulation of regulatory $\mathrm{T}$ cells in visceral adipose tissue of lean mice. Cell Metab. 2015; 21(4): 543-57.

11. Korn LL, Hubbeling HG, Porrett PM, Yang Q, Barnett LG, Laufer TM. Regulatory T cells occupy an isolated niche in the intestine that is antigen independent. Cell Rep. 2014; 9(5): 1567-73.

12. Chennupati V, Worbs T, Liu X, Malinarich FH, Schmitz S, Haas JD, et al. Intra- and intercompartmental movement of gammadelta $\mathrm{T}$ cells: intestinal intraepithelial and peripheral gammadelta $T$ cells represent exclusive nonoverlapping populations with distinct migration characteristics. J Immunol. 2010; 185(9): 5160-8.

13. Romagnoli PA, Sheridan BS, Pham QM, Lefrancois L, Khanna KM. IL-17A-producing resident memory gammadelta $T$ cells orchestrate the innate immune response to secondary oral Listeria monocytogenes infection. Proc Natl Acad Sci U S A. 2016; 113(30): 8502-7.

14. Lynch L, Michelet X, Zhang S, Brennan PJ, Moseman A, Lester C, et al. Regulatory iNKT cells lack expression of the transcription factor PLZF and control the homeostasis of T(reg) cells and macrophages in adipose tissue. Nat Immunol. 2015; 16(1): 85-95. 
15. Thomas SY, Scanlon ST, Griewank KG, Constantinides MG, Savage AK, Barr KA, et al. PLZF induces an intravascular surveillance program mediated by long-lived LFA-1-ICAM1 interactions. J Exp Med. 2011; 208(6): 1179-88.

16. Domiati-Saad R, Klintmalm GB, Netto G, Agura ED, Chinnakotla S, Smith DM. Acute graft versus host disease after liver transplantation: patterns of lymphocyte chimerism.

Am J Transplant. 2005; 5(12): 2968-73.

17. Metes D, Logar A, Rudert WA, Zeevi A, Woodward J, Demetris AJ, et al. Four-color flow cytometric analysis of peripheral blood donor cell chimerism. Hum Immunol. 2003; 64(8): 787-95.

*18. Stone JP, Mohamud M, Amin K, Critchley WR, Edge RJ, Clancy MJ, et al. Characterizing the early inflammatory contribution of the donor kidney following reperfusion. Nephrol Dial Transplant. 2017; 32(9): 1487-92.

Characterised lymphocytes and cytokines released from donor kidney following secondary preservation flush.

19. Fuchs EJ, Matzinger P. B cells turn off virgin but not memory T cells. Science. 1992; 258(5085): 1156-9.

20. Lassila O, Vainio O, Matzinger P. Can B cells turn on virgin T cells? Nature. 1988; 334(6179): 253-5.

21. Ronchese F, Hausmann B. B lymphocytes in vivo fail to prime naive T cells but can stimulate antigen-experienced T lymphocytes. J Exp Med. 1993; 177(3): 679-90.

22. Eynon EE, Parker DC. Small B cells as antigen-presenting cells in the induction of tolerance to soluble protein antigens. J Exp Med. 1992; 175(1): 131-8.

23. Schultze JL, Michalak S, Seamon MJ, Dranoff G, Jung K, Daley J, et al. CD40activated human $B$ cells: an alternative source of highly efficient antigen presenting cells to generate autologous antigen-specific $T$ cells for adoptive immunotherapy. J Clin Invest. 1997; 100(11): 2757-65.

24. von Bergwelt-Baildon MS, Vonderheide RH, Maecker B, Hirano N, Anderson KS, Butler MO, et al. Human primary and memory cytotoxic $\mathrm{T}$ lymphocyte responses are efficiently induced by means of CD40-activated B cells as antigen-presenting cells: potential for clinical application. Blood. 2002; 99(9): 3319-25.

25. Ise W, Inoue T, McLachlan JB, Kometani K, Kubo M, Okada T, et al. Memory B cells contribute to rapid Bcl6 expression by memory follicular helper T cells. Proc Natl Acad Sci U S A. 2014; 111(32): 11792-7.

26. Kurosaki T, Kometani K, Ise W. Memory B cells. Nat Rev Immunol. 2015; 15(3): 14959.

27. Leadbetter EA, Rifkin IR, Hohlbaum AM, Beaudette BC, Shlomchik MJ, MarshakRothstein A. Chromatin-IgG complexes activate B cells by dual engagement of IgM and Toll-like receptors. Nature. 2002; 416(6881): 603-7.

*28. Ali JM, Negus MC, Conlon TM, Harper IG, Qureshi MS, Motallebzadeh R, et al. Diversity of the CD4 T Cell Alloresponse: The Short and the Long of It. Cell Rep. 2016; 14(5): 1232-45.

Demonstrated using murine heart transplant model that depletion of donor $B$ cells or donor antigen presenting cells prior to heart graft retrieval reduced alloantibody responces in graft recipient

29. Nadarajah L, Ashman N, Thuraisingham R, Barber C, Allard S, Green L. Literature review of passenger lymphocyte syndrome following renal transplantation and two case reports. Am J Transplant. 2013; 13(6): 1594-600. 
30. Maxfield SJ, Taylor CJ, Kosmoliaptsis V, Broecker V, Watson CJ, Bradley JA, et al. Transfer of HLA-Specific Allosensitization From a Highly Sensitized Deceased Organ Donor to the Recipients of Each Kidney. Am J Transplant. 2015; 15(9): 2501-6.

31. Nouel A, Simon Q, Jamin C, Pers JO, Hillion S. Regulatory B Cells: An Exciting Target for Future Therapeutics in Transplantation. Front Immunol. 2014; 5: 11.

32. Newell KA, Asare A, Kirk AD, Gisler TD, Bourcier K, Suthanthiran M, et al. Identification of a B cell signature associated with renal transplant tolerance in humans. J Clin Invest. 2010; 120(6): 1836-47.

33. Shabir S, Girdlestone J, Briggs D, Kaul B, Smith H, Daga S, et al. Transitional B lymphocytes are associated with protection from kidney allograft rejection: a prospective study. Am J Transplant. 2015; 15(5): 1384-91.

34. Mauri C, Bosma A. Immune regulatory function of B cells. Annu Rev Immunol. 2012; 30: 221-41.

35. Deng S, Moore DJ, Huang X, Lian MM, Mohiuddin M, Velededeoglu E, et al. Cutting edge: transplant tolerance induced by anti-CD45RB requires $B$ lymphocytes. J Immunol. 2007; 178(10): 6028-32.

36. Rosser EC, Oleinika K, Tonon S, Doyle R, Bosma A, Carter NA, et al. Regulatory B cells are induced by gut microbiota-driven interleukin-1beta and interleukin- 6 production. Nat Med. 2014; 20(11): 1334-9.

37. Yoshizaki A, Miyagaki T, DiLillo DJ, Matsushita T, Horikawa M, Kountikov El, et al. Regulatory B cells control T-cell autoimmunity through IL-21-dependent cognate interactions. Nature. 2012; 491(7423): 264-8.

38. Baba $Y$, Matsumoto $M$, Kurosaki T. Signals controlling the development and activity of regulatory B-lineage cells. Int Immunol. 2015; 27(10): 487-93.

39. Prentice HG, Blacklock HA, Janossy G, Bradstock KF, Skeggs D, Goldstein G, et al. Use of anti-T-cell monoclonal antibody OKT3 to prevent acute graft-versus-host disease in allogeneic bone-marrow transplantation for acute leukaemia. Lancet. 1982; 1(8274): 7003.

40. Kataoka Y, Iwasaki T, Kuroiwa T, Seto Y, Iwata N, Hashimoto N, et al. The role of donor $\mathrm{T}$ cells for target organ injuries in acute and chronic graft-versus-host disease. Immunology. 2001; 103(3): 310-8.

41. Win TS, Rehakova S, Negus MC, Saeb-Parsy K, Goddard M, Conlon TM, et al. Donor CD4 $\mathrm{T}$ cells contribute to cardiac allograft vasculopathy by providing help for autoantibody production. Circ Heart Fail. 2009; 2(4): 361-9.

42. Motallebzadeh R, Rehakova S, Conlon TM, Win TS, Callaghan CJ, Goddard M, et al. Blocking lymphotoxin signaling abrogates the development of ectopic lymphoid tissue within cardiac allografts and inhibits effector antibody responses. FASEB J. 2012; 26(1): 5162.

43. Zhang $Q$, Reed EF. The importance of non-HLA antibodies in transplantation. Nat Rev Nephrol. 2016; 12(8): 484-95.

44. Win TS, Pettigrew GJ. Humoral Autoimmunity and Transplant Vasculopathy: When Allo Is Not Enough. Transplantation. 2010.

45. Cardinal H, Dieude M, Hebert MJ. The Emerging Importance of Non-HLA Autoantibodies in Kidney Transplant Complications. J Am Soc Nephrol. 2017; 28(2): 400-6. 46. Qureshi MS AJ, Chhabra M, et al. Germinal center humoral autoimmunity mediates progression of allograft vasculopathy independently from recipient alloimmunity.

Submitted. 
**47. Harper IG, Ali JM, Harper SJ, Wlodek E, Alsughayyir J, Negus MC, et al. Augmentation of Recipient Adaptive Alloimmunity by Donor Passenger Lymphocytes within the Transplant. Cell Rep. 2016; 15(6): 1214-27.

Investigated how graft versus host allorecogntion by donor CD4+ T cells augment recipient cellular and humoral alloimmune responses.

48. Hadad U, Martinez O, Krams SM. NK cells after transplantation: friend or foe. Immunol Res. 2014; 58(2-3): 259-67.

49. Kelly A, Trowsdale J. Introduction: MHC/KIR and governance of specificity. Immunogenetics. 2017; 69(8-9): 481-8.

50. van Bergen J, Thompson A, Haasnoot GW, Roodnat JI, de Fijter JW, Claas FH, et al. KIR-ligand mismatches are associated with reduced long-term graft survival in HLAcompatible kidney transplantation. Am J Transplant. 2011; 11(9): 1959-64.

51. van der Touw $W$, Bromberg JS. Natural killer cells and the immune response in solid organ transplantation. Am J Transplant. 2010; 10(6): 1354-8.

52. Fan X, Rudensky AY. Hallmarks of Tissue-Resident Lymphocytes. Cell. 2016; 164(6): 1198-211.

*53. Beura LK, Rosato PC, Masopust D. Implications of Resident Memory T Cells for Transplantation. Am J Transplant. 2016.

Reviewed donor-derived TRM cells as tolerance inducers for latent viral infections or as potential mediators of alloimmunogenecity.

54. Barbas AS, Knechtle SJ. Expanding the Donor Pool With Normothermic Ex Vivo Liver Perfusion: The Future Is Now. Am J Transplant. 2016; 16(11): 3075-6.

55. Smith CJ, Quinn M, Snyder CM. CMV-Specific CD8 T Cell Differentiation and Localization: Implications for Adoptive Therapies. Front Immunol. 2016; 7: 352.

56. Simon CO, Holtappels R, Tervo HM, Bohm V, Daubner T, Oehrlein-Karpi SA, et al. CD8 $\mathrm{T}$ cells control cytomegalovirus latency by epitope-specific sensing of transcriptional reactivation. J Virol. 2006; 80(21): 10436-56.

57. Khan TN, Mooster JL, Kilgore AM, Osborn JF, Nolz JC. Local antigen in nonlymphoid tissue promotes resident memory CD8+ $T$ cell formation during viral infection. J Exp Med. 2016; 213(6): 951-66.

58. Stern $M$, Hirsch $H$, Cusini A, van Delden $C$, Manuel $O$, Meylan $P$, et al. Cytomegalovirus serology and replication remain associated with solid organ graft rejection and graft loss in the era of prophylactic treatment. Transplantation. 2014; 98(9): 1013-8.

59. Burzyn D, Benoist C, Mathis D. Regulatory T cells in nonlymphoid tissues. Nat Immunol. 2013; 14(10): 1007-13.

60. Rosenblum MD, Gratz IK, Paw JS, Lee K, Marshak-Rothstein A, Abbas AK. Response to self antigen imprints regulatory memory in tissues. Nature. 2011; 480(7378): 538-42.

61. Malchow S, Leventhal DS, Nishi S, Fischer BI, Shen L, Paner GP, et al. Airedependent thymic development of tumor-associated regulatory T cells. Science. 2013; 339(6124): 1219-24.

62. Zhuang Q, Liu Q, Divito SJ, Zeng Q, Yatim KM, Hughes AD, et al. Graft-infiltrating host dendritic cells play a key role in organ transplant rejection. Nat Commun. 2016; 7: 12623.

63. Alegre ML, Lakkis FG, Morelli AE. Antigen Presentation in Transplantation. Trends Immunol. 2016; 37(12): 831-43. 
64. Zhang N, Schroppel B, Lal G, Jakubzick C, Mao X, Chen D, et al. Regulatory T cells sequentially migrate from inflamed tissues to draining lymph nodes to suppress the alloimmune response. Immunity. 2009; 30(3): 458-69.

65. Gagliani N, Jofra T, Valle A, Stabilini A, Morsiani C, Gregori S, et al. Transplant tolerance to pancreatic islets is initiated in the graft and sustained in the spleen. Am J Transplant. 2013; 13(8): 1963-75. 